Vol. 37 (1988) [89-92]

\title{
CONTINUOUS CHAOTIC FUNCTIONS OF AN INTERVAL HAVE GENERICALLY SMALL SCRAMBLED SETS
}

\author{
IVAN Mizera
}

\begin{abstract}
It is shown that continuous self-mappings of a compact interval, chaotic in the sense of $\mathrm{Li}$ and Yorke, have generically, in the uniform topology, only scrambled sets which are nowhere dense and of zero Lebesgue measure.
\end{abstract}

Let $f$ be a continuous function from a compact interval $I$ into itself. A set $S$ is called scrambled, if for every $x, y \in S, x \neq y$, and for every periodic point $p$ of $f$

$$
\begin{aligned}
& \limsup _{n \rightarrow \infty}\left|f^{n}(x)-f^{n}(y)\right|>0, \\
& \liminf _{n \rightarrow \infty}\left|f^{n}(x)-f^{n}(y)\right|=0, \\
& \limsup _{n \rightarrow \infty}\left|f^{n}(x)-f^{n}(p)\right|>0,
\end{aligned}
$$

where $f^{n}$ denotes the $n$-th iterate of $f$ (see [9]). A function $f$ is called chaotic in the sense of $\mathrm{Li}$ and Yorke when it has an uncountable scrambled set. This property is equivalent to the existence of a non-empty perfect "uniformly" scrambled set ([6], $[\mathbf{1 4}])$. The property "being chaotic" is generic $([\mathbf{1}],[\mathbf{2}],[\mathbf{8}],[\mathbf{1 1}])$. There are known examples of functions with scrambled sets of the first and of the second Baire category ([5]). If a scrambled set has the Baire property, it must be first category - hence it cannot be residual $([3],[4])$. A scrambled set can have a positive Lebesgue measure arbitrarily close to the measure of the whole interval $([\mathbf{7}],[\mathbf{1 2}],[\mathbf{1 3}])$. It can even have a full measure $([\mathbf{1 0}])$.

The size of the scrambled set - in the sense of the category or of the measure reflects somehow the degree of "chaos". However, in this paper we show that large scrambled sets are not typical for chaotic functions. We consider the space $C^{0}(I, I)$ of continuous functions of an interval into itself, endowed with the usual topology of uniform convergence. We prove the following

Received 6 March 1987

The author is grateful to Professor Jaroslav Smital, for introducing him to the subject, and to him and to Lubomir Snoha for valuable sugestions and remarks during the preparation of this paper.

Copyright Clearance Centre, Inc. Serial-fee code: 0004-9729/88 \$A2.00+0.00. 
ThEOREM. There is a first Baire category set $M \subseteq C^{0}(I, I)$ such that any $f \in$ $C^{0}(I, I) \backslash M$ has only scrambled sets (if any) which are nowhere dense in $I$ and of zero Lebesgue measure. In other words, mappings from $C^{0}(I, I)$ have generically only nowhere dense zero measure scrambled sets.

Combining the above mentioned results with the Theorem, we obtain

Corollary. Continuous self-mappings of an interval are generically chaotic but only with nowhere dense zero measure scrambled sets.

We prove the Theorem by several Lemmas. We may assume that $I=[0,1]$. Let $\lambda$ denote the Lebesgue measure on $I$. For $n=1,2,3, \ldots$, let $0<\zeta_{n}<4^{-n}$. For $i=1,2,3, \ldots, 2^{n}$ let $a(n, i)=(i-1) 2^{-n}, a\left(n, 2^{n}+1\right)=1, b(n, i)=i 2^{-n}-\zeta_{n}$. Let $I(n, i)=[a(n, i), b(n, i)], I_{n}=\bigcup_{i} I(n, i)$. Let $A_{n}=\left\{f \in C^{0}(I, I): f\left(I_{n}\right) \subseteq\right.$ int $\left.I_{n}\right\}$. Immediately we have

Lemma 1. For every $n, A_{n}$ is open in $C^{0}(I, I)$.

Let $B_{n}=\bigcup_{k \geqslant n} A_{k}$.

Lemma 2. For every $n, B_{n}$ is open and dense in $C^{0}(I, I)$.

Proof: By Lemma $1, B_{n}$ is open. Let $U_{\varepsilon}(f)$ be an $\varepsilon$-neighbourhood of $f$ in $C^{0}(I, I)$. By the uniform continuity of $f$ there exists an integer $k \geqslant n$ such that $2^{-k}<\epsilon / 4$ and

$$
|f(x)-f(y)|<\epsilon / 4 \text { whenever }|x-y| \leqslant 2^{-k} .
$$

Now we construct a function $g \in A_{k} \cap U_{e}(f)$ in two steps.

First define an auxiliary function $h: I_{k} \rightarrow I_{k}$ such that for every $i, h$ is constant on $I(k, i), h\left(I_{k}\right) \subseteq \operatorname{int} I_{k}$ and

$$
|h(a(k, i))-f(a(k, i))|<\varepsilon / 8 .
$$

We put $f(a(k, i))$ for the image of $I(k, i)$ under $h$, provided $f(a(k, i)) \in$ int $I_{k}$. Otherwise, we put for the image of $I(k, i)$ a slightly perturbed value: $f(a(k, i))+2^{-(k+1)}$ or $f(a(k, i))-2^{-(k+1)}$. Note that the gaps between the adjacent intervals of $I_{k}$ have each length $\zeta_{k}<4^{-k} \leqslant 2^{-(k+1)}$, while the length of each of these intervals is $2^{-k}-\zeta_{k}>$ $2^{-(k+1)}$. Hence, if $f(a(k, i)) \notin$ int $I_{k}$, then at least one of the perturbed values belongs to int $I_{k}$.

In the second step, let $g$ be a continuous extension of $h$ such that $g$ is linear on every interval contiguous to $I_{k}$ and $g(1)=f(1)$. Clearly $g \in A_{k}$, so it remains 
only to show that $|f(x)-g(x)|<\varepsilon$ for all $x \in I$. This is true for $x=1$; let $x \in[a(k, i), a(k, i+1))$ for some $i$. We have

$$
\begin{aligned}
& |f(x)-g(x)| \leqslant|f(x)-f(a(k, i))| \\
& +|f(a(k, i))-g(a(k, i))|+|g(a(k, i))-g(x)| .
\end{aligned}
$$

By (5), the middle term on the right of (6) is less than $\varepsilon / 8$. By the linearity of $g,(4)$ and (5) we get

$$
\begin{aligned}
& |g(a(k, i))-g(x)| \leqslant|g(a(k, i))-g(a(k, i+1))| \\
& \quad \leqslant \varepsilon / 8+|f(a(k, i))-f(a(k, i+1))|+\varepsilon / 8<\varepsilon / 2 .
\end{aligned}
$$

Now, summarizing all this, we have $|f(x)-g(x)|<\varepsilon$, and this completes the proof.

Lemma 3. Let $f \in A_{n}$, let $S$ be a scrambled set for $f$. If $S \cap I_{n} \neq \emptyset$, then

$$
\limsup _{k \rightarrow \infty}\left|f^{k}(u)-f^{k}(v)\right|<2^{-n} \quad \text { for all } u, v \in S
$$

Proof: Let $w \in S \cap I_{n}$. Then $f^{k}(w) \in \operatorname{int} I_{n}$ for every $k \geqslant 1$ since $f\left(I_{n}\right) \subseteq$ int $I_{n}$. Since actually $f\left(I_{n}\right) \subset$ int $I_{n}$, dist $\left\{f\left(I_{n}\right), I \backslash I_{n}\right\}>0$, hence (2) for $x=u$, $y=w$ implies that for any sufficiently large $k$ both $f^{k}(u)$ and $f^{k}(w)$ lie in the same component of $I_{n}$ (dependent on $k$ ). By the same argument applied to $v$ and $w$ we obtain that for sufficiently large $k$ both $f^{k}(u)$ and $f^{k}(v)$ lie in the same component of $I_{n}$, dependent on $k$. This implies (7).

Now we can complete the proof of the Theorem. Put $M=\bigcap_{n=1}^{\infty} B_{n}=$ $\bigcap_{n=1}^{\infty} \bigcup_{k=n}^{\infty} A_{k}$. By Lemma $2 M$ is residual in $C^{0}(I, I)$. Let $f \in M$, let $S$ be a scrambled set for $f$. There exists an infinite, increasing sequence $\left\{n_{i}\right\}_{i=1}^{\infty}$ of integers such that $f \in A_{n_{i}}$ for every $i$. By Lemma 3, there exists an integer $s$ such that $S \cap I_{n_{i}}=\emptyset$ for all $n_{i}>s$-since otherwise (1) would be violated. Let $J=\bigcup_{n_{i}>s}$ int $I_{n_{i}}$. For every $n, \lambda\left(I \backslash\right.$ int $\left.I_{n}\right)<2^{-n}$, hence $J$ has full measure. This implies $J$ is dense; clearly it is open. Since $S \subseteq I \backslash J$, we have that $\lambda(S)=0$ and $S$ is nowhere dense in I.

REMARK 1: We want to call attention to the following fact, implied by Lemmas 2 and 3. Let $M_{\varepsilon}$ be the set of all $f \in C^{0}(I, I)$ such that either all scrambled sets for $f$ have Lebesgue measure less than $\varepsilon$, or for every scrambled set $S$ (for $f$ ) is $\limsup _{n \rightarrow \infty}\left|f^{n}(x)-f^{n}(y)\right|<\varepsilon$ for all $x, y \in S$. Then $M_{\varepsilon}$ contains an open dense subset - its complement in $C^{0}(I, I)$ is nowhere dense. In other words, functions from $M_{e}$ are generically stable.

REMARK 2: The results admit a straight forward generalisation to the case of continuous self-mappings of the circle. They can be also extended to cover the $n$ dimensional case - for continuous functions of $I^{n}$ into $I^{n}$. 


\section{REFERENCES}

[1] L. Block, 'Stability of periodic orbits in the theorem of Šarkovskiī', Proc. Amer. Math. Soc. 81 (1981), 333-336.

[2] G.J. Butler, G. Pianigiani, 'Periodic points and chaotic functions in the unit interval', Bull. Austral. Math. Soc. 18 (1978), 255-265.

[3] A.M. Bruckner, Thakyin $\mathrm{Hu}$, 'On scrambled sets for chaotic functions', Trans. Amer. Math. Soc. 301 (1987), 289-297.

[4] T. Gedeon, 'There are no residual scrambled sets', Bull. Austral. Math. Soc. (to appear).

[5] K. Janková, 'On the stability of chaotic functions', preprint 1984.

[8] K. Janková, J. Smítal, 'A characterization of chaos', Bull. Austral. Math. Soc. 34 (1986), 283-292.

[7] I. Kan, 'A chaotic function possessing a scrambled set of positive Lebesgue measure', Proc. Amer. Math. Soc. 82 (1984), 45-49.

[8] P.E. Kloeden, 'Chaotic difference equations are dense', Bull. Austral. Math. Soc. 15 (1976), 371-379.

[9] T.Y. Li, J.A. Yorke, 'Period three implies chaos', Amer. Math. Monthly 82 (1975), 985-992.

[10] M. Misiurewicz, 'Chaos almost everywhere', in Iteration Theory and its Functional Equations: Lecture Notes in Mathematics 1163, ed. R.P. Liedl, L.P. Reich, G. Targonski (Springer, Berlin, 1985).

[11] M. Misiurewicz, 'Horseshoes for mappings of the interval', Bull. Acad. Polon. Sci. Sér. Sci. Math. 27 (1979), 167-169.

[12] J. Smital, 'A chaotic function with some extremal properties', Proc. Amer. Math. Soc. 87 (1983), 54-56.

[13] J. Smital, 'A chaotic function with a scrambled set of positive Lebesgue measure', Proc. Amer. Math. Soc. 82 (1984), 50-54.

[14] J. Smítal, 'Chaotic functions with zero topological entropy', Trans. Amer. Math. Soc. 287 (1986), 269-282.

Department of Mathematics

Komenský University

84215 Bratislava

Czechoslovakia 